\title{
Investigation of convection and diffusion during biodiesel production in packed membrane reactor using 3D simulation
}

\author{
Baharak Sajjadi ${ }^{a}$, A.R. Abdul Aziz ${ }^{a, *}$, Saeid Baroutian ${ }^{b}$, Shaliza Ibrahim ${ }^{a}$ \\ a Department of Chemical Engineering, Faculty of Engineering, University of Malaya, 50603 Kuala Lumpur, Malaysia \\ ${ }^{\mathrm{b}}$ SCION, Te Papa Tipu Innovation Park, 49 Sala Street, Private Bag 3020, Rotorua, New Zealand
}

\section{A R T I C L E I N F O}

\section{Article history:}

Received 7 February 2013

Accepted 24 July 2013

Available online xxx

\section{Keywords:}

Transesterification

Membrane reactor

Computational fluid dynamics (CFD)

Brinkman equations

Stephan-Maxwell equation

\begin{abstract}
A B S T R A C T
The 3D simulation of convection and diffusion phenomena within a ceramic membrane during transesterification reaction was the aim of this study. The ceramic membrane was a tubular micro porous $\mathrm{TiO}_{2} / \mathrm{Al}_{2} \mathrm{O}_{3}$ packed with the heterogeneous catalyst. The Navier-Stokes, Brinkman and StephanMaxwell equations were applied for investigation of fluid flow reaction and mass transfer within the system. The value of the convection was generally between $10^{4}$ and $10^{7}$ times higher than diffusion. It depends on concentration component, the diffusion coefficient and components velocity. A good agreement was found with the maximum deviation of $8 \%$ from experimental data.
\end{abstract}

(c) 2013 The Korean Society of Industrial and Engineering Chemistry. Published by Elsevier B.V. All rights reserved.

\section{Introduction}

Biodiesel (fatty acid methyl ester) is considered as a cleanburning fuel which is produced from renewable sources [1]. It has excellent lubricating properties and does not contain any aromatic hydrocarbons, metals, sulphur or crude oil residues. Thus, it is a good alternative for petroleum based diesel because it reduces volatile organic compounds emissions (VOC) and greenhouse gases (GHG). It is much safer than mineral diesel because of its higher flash point. Furthermore, it has a high capacity of self-ignition because of its high cetane number [2]. Transesterification is the most common process for fatty acid methyl ester (FAME) production. It includes three chain reactions. In each step, one molecule of alcohol reacts with one molecule of glyceride (tri, di or mono) and produces one molecule of alkyl ester. Diglyceride (DG) and mono-glyceride (MG) are intermediate products which converted to glycerol and alkyl ester during the reaction [3]. These chain reactions assist by enzymatic, homogeneous or heterogeneous catalysts [4]. Catalyst free transesterification is another technique. However it requires a high temperature $\left(>350^{\circ} \mathrm{C}\right.$ ) and pressure (20 MPa) [5].

The enzymatic catalysts give a high conversion rate and are selective. Besides, their productions can be separated easily. But,

\footnotetext{
* Corresponding author. Tel.: +60 379675300; fax: +60 379675319.

E-mail addresses: azizraman@um.edu.my,rshazrin@gmail.com (A.R. Abdul Aziz).
}

those which show unstable activities and require long reaction time, also are not economical yet [6]. Homogeneous (acidic or alkaline) catalyzed processes also suffer from some weaknesses such as high cost of purification and wastewater generation for final removal of the catalyst. In acidic homogeneous transesterification, increase in the reaction time, temperature and the corrosive nature of the catalyst are the other problems. More sensitivity to the purity of the reactant is also reported in case of alkaline catalyzed reaction. Heterogeneous catalyst can overcome some of the mentioned problems, because it can be easily removed by filtration and it is non-corrosive. The only problem is the spread of the catalyst which is due to providing an enough space for active site for production. For this purpose several techniques have been applied to increase the constitution of the three-phase system (triglycerides, alcohol and solid catalyst). The most preferred techniques are those which support the catalysts on non-reaction materials, or use them in porous media [7]. Using membrane is the newest technology which has been applied for both production and purification of FAME. This method can concentrate on process streams, recover valuable products and improve the conversion rate of the reaction. The selectivity of the membrane is the other advantage of this method which depends on its pore sizes. Also, their special physical and chemical consistency makes their function reproducible in all over of their life time [8]. Therefore, membrane bioreactors can be suitable alternatives to produce biodiesel due to its ability to overcome some limitations during production and purification. The high-quality biodiesel production of FAME via this technique (more than $99 \%$ ) has been also reported 


\section{Nomenclature}

Symbols

$\mathrm{CV} \quad$ control volume $\left(\mathrm{m}^{3}\right)$

C component concentration $\left(\mathrm{mol} / \mathrm{m}^{3}\right)$

$D_{i j} \quad$ Fick diffusivity $\left(\mathrm{m}^{2} / \mathrm{s}\right)$

$D_{i}^{T} \quad$ thermal diffusion coefficient $(\mathrm{kg} / \mathrm{m} \mathrm{s})$

div divergence

E rate-of-strain tensor

$F \quad$ acting force (N)

$K \quad$ kinetic rate constant $\left(\mathrm{dm}^{3} / \mathrm{mol} \mathrm{min}\right)$

M molecular mass $(\mathrm{g} / \mathrm{mol})$

$n$ normal vector

$t$ time (s)

$T$ temperature $\left({ }^{\circ} \mathrm{C}\right)$

$T$ deviatoric stress tensor

$u \quad$ velocity vector $(\mathrm{m} / \mathrm{s})$

$V \quad$ volume $\left(\mathrm{m}^{3}\right)$

$w \quad$ weight, various

$p \quad$ pressure $(\mathrm{Pa})$

$x_{j} \quad$ molar fraction of species $(j)$

$R_{i} \quad$ reaction rate $\left(\mathrm{kg} / \mathrm{m}^{3} \mathrm{~s}\right)$

$Q_{b r} \quad$ mass sink source $\left(\mathrm{kg} / \mathrm{m}^{3} \mathrm{~s}\right)$

\section{Greek symbols}

$\rho \quad$ density of the mixture $\left(\mathrm{kg} / \mathrm{m}^{3}\right)$

$\mu \quad$ molecular viscosity $\left(\mathrm{Ns} / \mathrm{m}^{2}\right)$

$S_{\phi} \quad$ source-sink term

$\varepsilon \quad$ fractional hold up of each component

$\varepsilon_{p} \quad$ porosity

$\kappa \quad$ permeability $\left(\mathrm{m}^{2}\right)$

$\beta_{f} \quad$ Forchheimer coefficient $\left(\mathrm{kg} / \mathrm{m}^{4}\right)$, for considering of the drag of the fluid on the porous matrix $\left(\mathrm{kg} / \mathrm{m}^{4}\right)$

$\omega_{i} \quad$ mass fraction of species $i$

$\Gamma \quad$ appropriate coefficient for variable $\varepsilon$ (here is the diffusion constant)

$S_{\phi} \quad$ source-sink term per unit volume

d gradient

\section{Subscripts}

$\mathrm{MeOH}$ methanol

TG triglyceride

DG diglyceride

MG monoglyceride

G glyceride

FAME fatty acid methyl ester

RBD refined, bleached and deodorized

by some authors [2,9-11]. But, to better comprehend the design, control and optimization of the process we require the knowledge of membrane phenomena such as convection and diffusion.

The relative complexity of fluid flow in membrane systems or thin channels surrounded by permeable walls highlights the necessity of understanding the underlying problems.

The study of convection and diffusion in such systems is accompanied by some challenges. These two mechanisms are affected by fluid flow rates, membrane surface area, temperature and the membrane thickness.

Generally, there are two main models which make it possible to understand the mathematics and physics of the membrane phenomenon. The first model is based on the concept of capillarity. It is a solubilization-diffusion model which is developed by Kadem and Katchalsky [12]. The second one is the model of polarization that results in a progressive accumulation of species (molecules, particles, etc.) stopped on the surface of the membrane [13]. The complexity of these models decreases by CFD simulations. These numerical simulations help us to minimize the number of experiments and better comprehend the processes in a shorter time. However, the numerical simulation of fluid flow and transport in membrane systems is a challenging problem; because these are composed of porous materials and contain fractures and cavities on multiple scales. On micro scale, which fluid flows at low Reynold's numbers through pore throats and individual pores, incompressible Navier-Stokes equation is applicable for description of situation. But on macro scale, flow should be described by set of effective petro physical parameters such as porosity and permeability. These parameters describe the average ability of the membrane to store or transport fluids. Then the flow should be modeled using Darcy's law along with the Navier-Stokes and mass conservation. In Stokes-Darcy approach, Darcy's law is applicable for porous material and the Stokes equation for the voids. The boundaries between the porous and void volumes can be described by the Beavers-Joseph-Saffman method [14]. Stokes-Brinkman is another approach which gives a seamless transition between the Darcy and Stokes equations. In this model, a new term is added to the Stokes equations that accounts for viscous transport in momentum balance. Solving either Stokes-Brinkman or DarcyStokes equations in 3D systems because of its computational cost is not applicable even in a high-performance supercomputer. Therefore, well parallelizable iterative solvers are usually employed. In this technique, the number of iterations converges to a satisfactory solution with the least error which depends on the condition number of the matrix system.

In this study, we focused on the simulation of a tubular ceramic $\left(\mathrm{TiO}_{2} / \mathrm{Al}_{2} \mathrm{O}_{3}\right)$ membrane which was used for biodiesel production. The ceramic membrane was packed with potassium hydroxide supported on activated carbon as the heterogeneous catalytic bed. A set of 3D model using finite element method (FEM) was considered for time dependent simulation of the mentioned system. The transport equations were described by Navier-Stokes for fluid flow, Brinkman equations for porous media and StephanMaxwell equations for conversion rate of reaction and convectiondiffusion mechanisms. Besides, the PARDISO algorithm was applied to combine and solve the equations. This algorithm is a direct sparse solver which supports parallel processing. The numerical discretization and analysis schemes were then validated with experimental results of velocity distribution and reaction yield.

\section{Experimental setup}

The experimental data has been taken from the work of Baroutian et al, and it is summarized briefly for easier understanding of readers [15]. Fig. 1 shows the schematic diagram of experimental setup of biodiesel production. The membrane reactor consisted of a shell and a tube with one feed inlet and two outlets. One outlet is the end of the catalytic bed and the other is the ceramic membrane body. Commercial ceramic membrane (Atech Innovations $\mathrm{GmbH}$, Germany) was used as both catalytic bed and separator. This system was covered by another shell which only used for gathering the production. The membrane was a porous media of $\mathrm{TiO}_{2} / \mathrm{Al}_{2} \mathrm{O}_{3}$ with the pore sizes of $0.05 \mu \mathrm{m}$ and the filtration surface of $0.0201 \mathrm{~m}^{2}$. The length and the diameters of the inner and outer of the membrane were $40 \mathrm{~cm}, 1.60 \mathrm{~cm}$ and $2.54 \mathrm{~cm}$, respectively. Both ends of the ceramic tube were enameled in order to keep the inside separate from the outside 


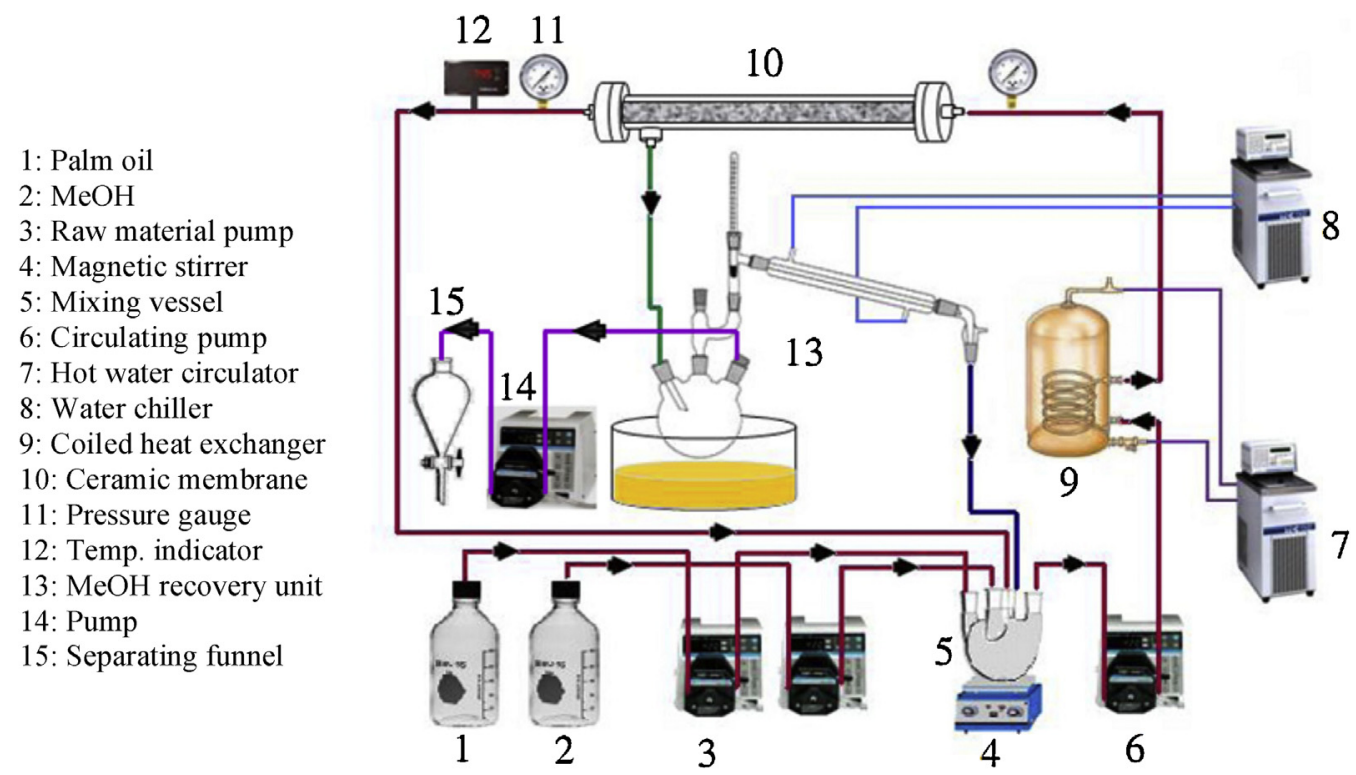

Fig. 1. Schematic diagram of packed bed membrane reactor to produce biodiesel [15].

Table 1

Experimental design and the response value.

\begin{tabular}{|c|c|c|c|}
\hline Temperature $\left({ }^{\circ} \mathrm{C}\right)$ & Mass of catalyst per unit volume of reactor $\left(\mathrm{mg} / \mathrm{cm}^{3}\right)$ & Cross flow circulation velocity $(\mathrm{cm} / \mathrm{s})$ & Conversion \\
\hline 50 & 143.75 & 0.195 & 87.5 \\
\hline 60 & 143.75 & 0.212 & 92.4 \\
\hline
\end{tabular}

during the activity tests. The tube region was packed with heterogeneous catalyst which occupied $50 \%$ of the inner section. The heterogeneous catalyst is kept in stable places using stainless steel screens attached to the upstream and downstream tubing. The characterisation of catalytic bed has shown in Table 2. Then, uniform distribution of catalyst was assumed in CFD section. Palm oil and methanol with the volume ratio of $1: 1$ were charged into the system after reaching a stable reaction temperature (50 and $\left.60{ }^{\circ} \mathrm{C}\right)$.

RBD palm oil with the iodine value of 53.2, the acid value of 0.5 and the water mass fraction of $400 \times 10^{-6}$ and methanol with the purity of $99.8 \%$ were used as the initial reactants. Pure potassium hydroxide $(98.9 \%)$ was used as a catalyst for transesterification. Palm shell-based activated carbon in this work was produced by physical activation process using steam as the activating agent. The solution of potassium was prepared by dissolving potassium hydroxide in deionized water. The potassium hydroxide solutions and the activated carbon were then agitated together for $24 \mathrm{~h}$ in an orbital shaker with the temperature of $25^{\circ} \mathrm{C}$ and the constant circulation of $180 \mathrm{rpm}$. The prepared heterogeneous catalyst contacting potassium hydroxide on activated carbon was sieved, washed with deionized, dried with oven and then cooled and stored.

The pressure and the temperature of the system during the reaction were monitored by pressure gauges and temperature indicator. The conversion rate of the reaction was calculated via the difference between the initial and the remaining mass of the triglycerides. Due to the small molecular size, methanol molecules were able to pass through the membrane along with the biodiesel and glycerol. The values of the reaction rate constants which exactly matched with this work were obtained from available literature (Table 3) [16]. Two different runs were selected for CFD simulation according to the available kinetics information (Table 1).

\section{Theoretical study}

\subsection{The simulated system}

In Fig. 2, different layers of catalytic bed (inner layer), ceramic membrane (intermediate layer) and storage system (outer layer) are shown. In Fig. 3, the simulated layers are illustrated. In order to decrease the memorial computational demand, two simplifications in design were done. First, the third layer that was used for the collection of products was neglected. Since the flow is axisymmetric, only half of the system was studied. All the boundary conditions are presented in Fig. 3. For initiating the numerical solution, the velocity and the component concentrations were specified at the inlet. At all the other locations the values were taken to be zero at $t=0$.

\subsection{The simplification assumptions}

In order to simulate the membrane system using Brinkman equation, the problem should be simplified using several assumptions. In the first assumption, fluctuation of temperature profile within the catalytic bed and ceramic membrane was ignored. This is due to the complexity of combined equations and high freedom degree of this 3D system. In other words, most of the

Table 2

Characterization results for the catalytic bed [15].

\begin{tabular}{lcll}
\hline Property & Value & Unit & Technique \\
\hline BET surface area & 214.46 & $\mathrm{~m}^{2} / \mathrm{g}$ & BET \\
Pore volume & 0.174 & $\mathrm{~cm}^{3} / \mathrm{g}$ & BET \\
Micro pore volume & 0.114 & $\mathrm{~cm}^{3} / \mathrm{g}$ & BET \\
Average pore width & 3.06 & $\mathrm{~nm}$ & BET \\
Active sites concentration & 1.558 & $\mathrm{mmol} / \mathrm{g}$ & TPD-CO2
\end{tabular}




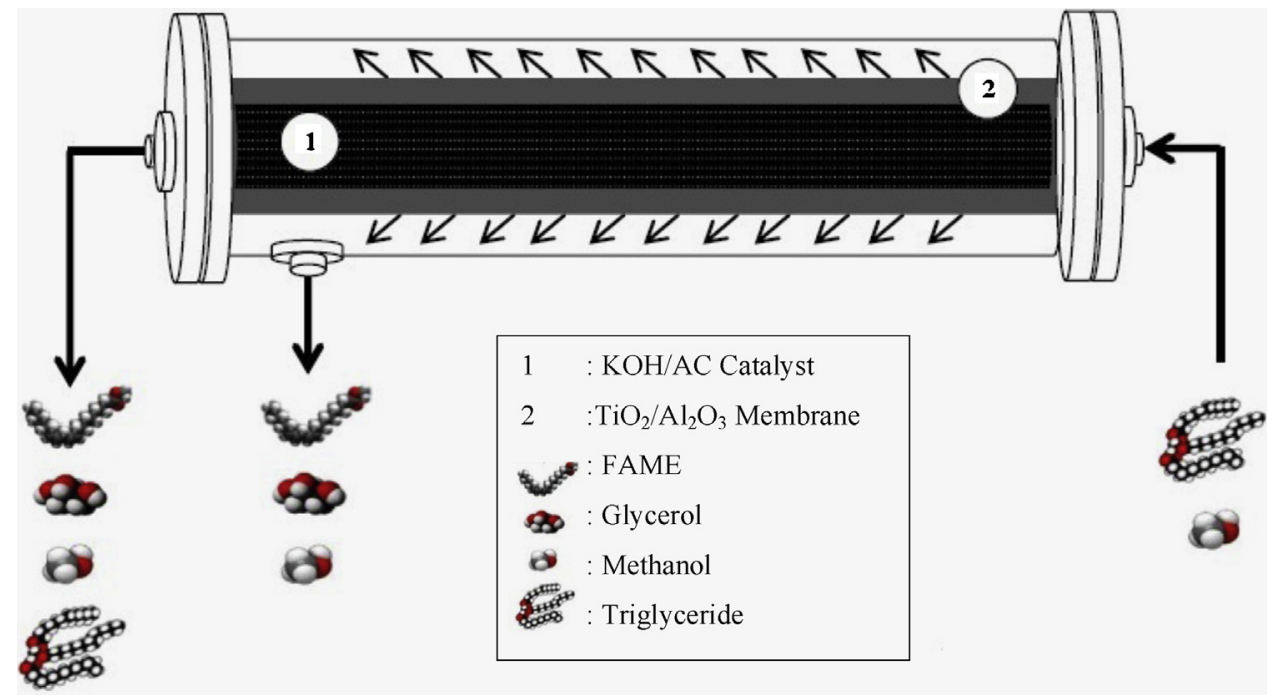

Fig. 2. Combination of heterogeneous base transesterification and triglyceride separation in the packed bed membrane reactor.

physical parameters such as density, viscosity and diffusion coefficient are dependent on temperature. By considering the role of temperature, a new variable was added to all equations instead of each constant parameter. This variable depends on the time, so the complexity of the system and the freedom degree could increase severely and even high performance supercomputers cannot solve the equations. In the second assumption, we considered the fluid as an incompressible Newtonian flow. Since the Re number is sufficiently small, fully developed velocity profile at the catalytic bed entrance was assumed. This assumption is indicated by Fig. 5. Chemical reaction was considered only at the surface of the catalyst and diffusion of the material within the catalysts was ignored. Since there is no catalyst within ceramic membrane, no reaction was assumed in this section. Due to the high packing density of the ceramic membrane, the permeability was estimated to be isotropic.

Finally, in this study, the catalysts particles were packed inside the ceramic membrane and held in place using stainless steel screens attached to the down and upstream tubing. This technique was used for the prevention of axisymmetric accumulation of catalysts particles, generation of a uniform disperse of catalyst and well mixing of methanol with TG. Thus the assumption of single phase approached was applied which is matched with the experiments.

\section{Governing equations for the fluid flow}

In fluid dynamics, the continuity and momentum balance equations are usually solved to obtain the flow field [17].

$\overbrace{\frac{\partial(\rho \varepsilon)}{\partial t}}^{\text {unsteady term }}+\overbrace{\operatorname{div}(\rho \vec{u} \varepsilon)}^{\text {convection term }}=\overbrace{\operatorname{div}\left(\Gamma_{\varepsilon} \text { grad } \varepsilon\right)}^{\text {dif fusion term }}+\overbrace{S_{\phi}}^{\text {source term }}$

Since the liquid modeling is incompressible, the governing equation in the fluid region was given by incompressible NavierStokes equation. But, ceramic membrane is a porous media and Navier-Stokes cannot explain the fluid flow within this system. This means that it is a solid matrix with an interconnected pores (or voids) which allows the flow of one or more fluids to pass through the matrix. This situation should be considered in Navier-Stokes equation via Darcy, Brinkman or the other methods. In this study the Brinkman equation was used to modify the Navier-Stokes equation. Besides, we used the Maxwell-Stephan diffusion and convection equation at transient situation to investigate the mass-balance within the catalytic bed and ceramic membrane. All the mentioned equations are summarized in Table 5.

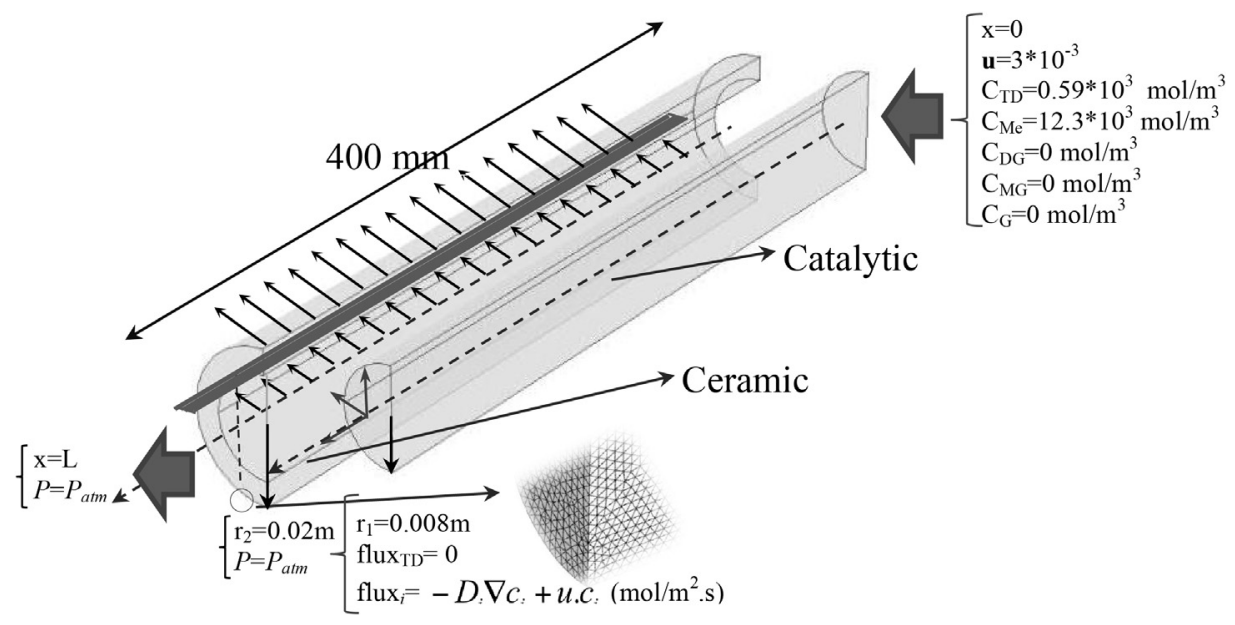

Fig. 3. Reactor different sections attached with the boundary conditions at different surfaces. 


\subsection{Discretization of Brinkman equation by the finite element method}

In Darcy method, it is assumed that velocity is proportional to the pressure gradient for a particular porous media. Finally, for the saturated flow in porous media the equation below is suggested.

$\frac{\mu}{k_{b r}} \vec{u}-\nabla p=0$

$\nabla \cdot \vec{u}=0$

which $k$ denoted as permeability tensor. This model does not take into account the convective acceleration and viscous resistance terms. Also, Darcy's linear relationship between the pressure gradient and discharge velocity breaks down at higher porosity. That situation is considered by Brinkman's law which is the extended form of Darcy's law by addition of a viscous term. In Brinkman equation, the directional velocities and pressure are dependent variables.

$\mu \nabla \vec{u}-\nabla p-\frac{\mu}{k_{b r}} \vec{u}=0$

In incompressible fluid, the flow should satisfy $\operatorname{div} \vec{u}=0$, thus the stress tensor is written as

$T=\sigma^{d}=p I$

where $\sigma^{d}$ is deviatoric stress and can be defined by:

$\sigma^{d}=2 \mu \nabla^{s} \vec{u}$

where $\nabla^{s} \vec{u}$ defined the strain rate and $\mu$ denotes the viscosity of the fluid. By the mentioned assumption (Section 3.2), a usual form of Brinkman equation in equilibrium situation is defined by:

$\operatorname{div} \sigma^{d}-\nabla p-c \vec{u}=0$

where $\vec{u}$ and $p$ are the variables to be solved, $c$ is constant value $\left(c=\mu_{e} / k\right), \mu_{e}$ is the dynamic viscosity and $k$ is the permeability. Permeability value explains the homogenized behavior of the porous medium. However in the scope of a full detailed simulation it would lead to billions of freedom degrees within the simulation.

In order to use the finite element method for numerical simulation of Brinkman equation, it should change to weak formulation. By integration of Brinkman equation and using the test function of $\vec{u}^{*}$, Eq. (7) could be written as:

$\forall \vec{u}^{*}: \int_{\Omega}\left(\operatorname{div} \sigma^{d} \cdot \vec{u}^{*}-\nabla p \cdot \vec{u}^{*}+c \vec{u} \cdot \vec{u}^{*}\right) d \Omega=0$

After using the divergence theorem, the first term of Eq. (8) can be calculated by:

$$
\begin{gathered}
\forall \vec{u}^{*}: \int_{\Omega} \operatorname{div}\left(\sigma^{d} \cdot \vec{u}^{*}\right) d \Omega=\int_{\partial \Omega} \sigma^{d} \cdot \vec{n} \cdot \vec{u}^{*} d \partial \Omega \\
=\int_{\Omega} \operatorname{div} \sigma^{d} \cdot \vec{u}^{*} d \Omega+\int_{\Omega} \sigma^{d}: \nabla \vec{u}^{*} d \Omega
\end{gathered}
$$

And the second term of Eq. (8) can be calculated by:

$$
\begin{aligned}
\forall \vec{u}^{*}: \mid \int_{\Omega} \operatorname{div}\left(p \cdot \vec{u}^{*}\right) d \Omega=\int_{\partial \Omega} p \cdot \vec{u}^{*} \cdot \vec{n} d \partial \Omega= & \int_{\Omega} \nabla p \cdot \vec{u}^{*} d \Omega \\
& +\int_{\Omega} p \nabla \vec{u}^{*} d \Omega
\end{aligned}
$$

Finally, Eq. (11) is the result of the mentioned mathematical steps.

$$
\begin{aligned}
\forall \vec{u}^{*} & : \int_{\Omega}\left(\sigma^{d}\right. \\
& \left.: \nabla \vec{u}^{*}-p \nabla \vec{u}^{*}\right) d \Omega+\int_{\partial \Omega}\left(\sigma^{d} \cdot \vec{n} \cdot \nabla \vec{u}^{*}-p \vec{n} \cdot \vec{u}^{*}\right) d \partial \Omega=0
\end{aligned}
$$

By using the total stress tensor of $T=\sigma^{d}-p I$ and $\vec{t}=T \cdot \vec{n}$, Eq. (11) can be rewritten as

$\forall \vec{u}^{*}: \int_{\Omega}\left(\sigma^{d}: \nabla \vec{u}^{*}-p \cdot \nabla \vec{u}^{*}\right) d \Omega+\int_{\partial \Omega}\left(\vec{t} \cdot \vec{u}^{*}\right) d \partial \Omega=0$

By imposing the test function of pressure $p^{*}$, the weak formulation of mass conservation can be written as:

$\forall p^{*}:-\int_{\Omega}\left(p^{*} \cdot \operatorname{div} \vec{u}\right) d \Omega=0$

By combining the Eqs. (12) and (13) and noticing $\sigma^{d}-p I=T$, the mixed formulation can be simplified to

$$
\begin{aligned}
\forall\left(\vec{u}^{*}, p^{*}\right) & : \int_{\Omega} T: \nabla \vec{u}^{*} d \Omega-\int_{\partial \Omega}\left(\vec{t} \cdot \vec{u}^{*}\right) d \partial \Omega-\int_{\Omega}\left(P^{*} \operatorname{div} \vec{u}^{*}\right) d \Omega \\
& +\int_{\Omega} C \vec{u} \vec{u}^{*} d \Omega=0
\end{aligned}
$$

where $\vec{u}^{*}$ and $p^{*}$ are the test functions and can be defined by $\vec{u}^{*}=\left\{\vec{u}_{\text {node }}^{*}\right\}\left[N_{v}\right]$ and $p^{*}=\left\{p_{\text {node }}^{*}\right\}\left[N_{p}\right]$ in these equations, $\left[N_{v}\right]$ and $\left[N_{p}\right]$ are the shape functions of the velocity and the pressure, respectively. Besides, $\left\{\vec{u}_{\text {node }}^{*}\right\}$ and $\left\{p_{\text {node }}^{*}\right\}$ are the values at the node for test velocity and test pressure. By interpolate the mentioned parameters, Brinkman equation can be written as

$$
\begin{gathered}
\forall\left(\vec{u}^{*}, p^{*}\right): \int_{\Omega}\left\{\left\{\nabla \vec{u}_{\text {node }}^{*}\right\} \nabla\left[N_{v}\right]\right\} d \Omega-\int_{\partial \Omega} \vec{t} \cdot\left\{\left\{\vec{u}_{\text {node }}^{*}\right\} \nabla\left[N_{v}\right]\right\} d \partial \Omega \\
-\int_{\Omega}\left\{p_{\text {node }}^{*}\right\}\left[N_{p}\right] \operatorname{div} \vec{u} \Omega+\int_{\Omega} c \vec{u} \cdot\left\{\vec{u}_{\text {node }}^{*}\right\}\left[N_{v}\right] d \Omega=0
\end{gathered}
$$

By calculation the derivations over the $\vec{u}_{\text {node }}^{*}$ and $p_{\text {node }}^{*}$, the test function of velocity and pressure will be eliminated and the equation will be a function of velocity and pressure by:

$$
\begin{aligned}
R(\vec{u}, p)= & \int_{\Omega} T:\left\{\left\{\nabla \vec{u}_{\text {node }}^{*}\right\} \nabla\left[N_{v}\right]\right\} d \Omega-\int_{\partial \Omega} \vec{t} \cdot\left\{\left\{\vec{u}_{\text {node }}^{*}\right\} \nabla\left[N_{v}\right]\right\} d \partial \Omega \\
& -\int_{\Omega}\left\{p_{\text {node }}^{*}\right\}\left[N_{p}\right] \operatorname{div} \vec{u} \Omega+\int_{\Omega} c \vec{u} \cdot\left\{\vec{u}_{\text {node }}^{*}\right\}\left[N_{v}\right] d \Omega=0
\end{aligned}
$$

Thus the derivation of function $R(\vec{u}, p)$ (3.6) over $\vec{u}_{\text {node }}^{*}$ and $p_{\text {node }}^{*}$

$$
\vec{r}\left[\begin{array}{c}
\frac{\partial R}{\partial\left\{\vec{u}_{\text {node }}^{*}\right\}} \\
\frac{\partial R}{\partial\left\{p_{\text {node }}^{*}\right\}}
\end{array}\right]=\int_{\Omega}\left[\begin{array}{l}
T: \nabla\left[N_{v}\right]+c\left(\vec{u}-\vec{u}_{\text {imp }}\right) \cdot\left[N_{v}\right] \\
-\left[N_{p}\right] \cdot \operatorname{div} \vec{u} d \Omega
\end{array}\right]-\int_{\Omega}\left[\begin{array}{l}
\vec{t} \cdot\left[N_{v}\right] \\
0
\end{array}\right]
$$

Combination of the continuity equation and the momentum balance equation using Brinkman equation together brings about the fluid flow in this work.

The validity of the Darcy-Brinkman approach has been confirmed by several investigations in relation to the boundary conditions at the fluid as well as solid-interface [18]. The important aspect of the Brinkman method is to introduce penalty terms into Navier-Stokes equations results in additional stiffness. Then, the use of stiffly stable solvers or implicit treatment is required for the penalization terms [19].

4.2. Discretization of convection-diffusion equation by the finite element method

$\frac{\partial c_{i}}{\partial r}+\nabla \cdot\left(-D_{i}\left(\frac{\partial c_{i}}{\partial x}+\frac{\partial c_{i}}{\partial y}+\frac{\partial c_{i}}{\partial z}\right)\right)+u\left(\frac{\partial c_{i}}{\partial x}+\frac{\partial c_{i}}{\partial y}+\frac{\partial c_{i}}{\partial z}\right)+\beta c_{i}=R_{i}$

$N_{i}=-D\left(\frac{\partial c_{i}}{\partial x}+\frac{\partial c_{i}}{\partial y}+\frac{\partial c_{i}}{\partial z}\right)+u \cdot c_{i}$ 
Table 3

Reaction rate constant $k$ (wt\% $\mathrm{min})^{-1}$ for triglyceride (TG), diglyceride (DG), and monoglyceride (MG) at different temperatures [16].

\begin{tabular}{|c|c|c|c|}
\hline Reaction & Temperature $\left({ }^{\circ} \mathrm{C}\right)$ & Reaction rate constant $(\mathrm{wt} \% \mathrm{~min})^{-1}$ & $R^{2}$ \\
\hline $\mathrm{TG}+\mathrm{CH}_{3} \mathrm{OH} \rightarrow \mathrm{DG}+\mathrm{R}_{1} \mathrm{COOCH}_{3}$ & $\begin{array}{l}50 \\
60\end{array}$ & $\begin{array}{l}0.018 \\
0.036\end{array}$ & $\begin{array}{l}0.9865 \\
0.9822\end{array}$ \\
\hline $\mathrm{DG}+\mathrm{CH}_{3} \mathrm{OH} \rightarrow \mathrm{MG}+\mathrm{R}_{2} \mathrm{COOCH}_{3}$ & $\begin{array}{l}50 \\
60\end{array}$ & $\begin{array}{l}0.036 \\
0.070\end{array}$ & $\begin{array}{l}0.9940 \\
0.9860\end{array}$ \\
\hline $\mathrm{MG}+\mathrm{CH}_{3} \mathrm{OH} \rightarrow \mathrm{MG}+\mathrm{R}_{3} \mathrm{COOCH}_{3}$ & $\begin{array}{l}50 \\
60\end{array}$ & $\begin{array}{l}0.112 \\
0.141\end{array}$ & $\begin{array}{l}0.9733 \\
0.9843\end{array}$ \\
\hline
\end{tabular}

Table 4

Diffusion coefficient $D\left(\mathrm{~m}^{2} / \mathrm{s}\right)$ of reactants and products.

\begin{tabular}{|c|c|c|c|}
\hline Components & Temperature $\left({ }^{\circ} \mathrm{C}\right)$ & Diffusion coefficient & References \\
\hline Triglyceride (TG) & 60 & $1 \times 10^{-13}$ & {$[24]$} \\
\hline Diglyceride (DG) & 60 & $1.55 \times 10^{-13}$ & [24] \\
\hline \multirow[t]{2}{*}{ Monoglyceride (MG) } & 27 & $1 \times 10^{-12}$ & [25] \\
\hline & 87 & $3 \times 10^{-12}$ & \\
\hline Glycerol & 40 & $2 \times 10^{-10}$ & {$[26]$} \\
\hline Fatty acid methyl ester & 60 & $1 \times 10^{-11}$ & [27] \\
\hline Methanol & 60 & $4.8 \times 10^{-12}$ & [28] \\
\hline
\end{tabular}

where $u$ and $D$ denote the velocity and components diffusion coefficient (Table 4), respectively.

$\nabla \cdot\left[\rho \omega_{i} u-\rho \omega_{i} \sum_{j=1}^{n} \tilde{D}_{i j}\left(\nabla x_{j}+\left(x_{j}-\omega_{j}\right) \frac{\nabla p}{p}\right)-D_{i}^{T} \frac{\nabla T}{T}\right]=R_{i}$

In the stationary case, Eq. (18) reduces to:

$-\operatorname{div}(D \nabla c)+(u \cdot \nabla c)+\beta c=R$
To generate a unique solution, it is necessary to define exactly an initial condition and one boundary condition at each part of the boundary. In order to discretize the convection-diffusion equation by finite elements standard Galerkin and upwinding technique were used. After integration over the domain yields and by a timeindependent test function $\vec{u}^{*}$, Eq. (22) can be written as:

$\int_{\Omega} \rho \frac{\partial c}{\partial t} \vec{u}^{*} d \Omega+\int_{\Omega}\{-\operatorname{div}(D \nabla c)+(u \cdot \nabla c)+\beta c\} \vec{u}^{*} d \Omega=\int_{\Omega} R \vec{u}^{*} d \Omega$

Table 5

Governing equations for a Newtonian incompressible fluid in cylindrical coordinates.

The continuity equation

$\overbrace{\frac{\partial(\rho \varepsilon)}{\partial t}}^{\text {unsteady term }}+\overbrace{\operatorname{div}(\rho \vec{u} \varepsilon)}^{\text {convection term }}=\overbrace{\operatorname{div}\left(\Gamma_{\varepsilon} \text { grad } \varepsilon\right)}^{\text {dif fusion term }}+\overbrace{S_{\phi}}^{\text {source term }}$

Brinkman equation in radial direction

$\frac{\rho}{\varepsilon_{p}}\left(\frac{\partial u_{x}}{\partial t}+u_{x} \frac{\partial u_{x}}{\partial x}+u_{y} \frac{\partial u_{x}}{\partial y}+u_{z} \frac{\partial u_{z}}{\partial z}\right)=\left[-\frac{\partial p}{\partial x}+\frac{\mu}{\varepsilon_{p}}\left(\left(\frac{\partial^{2} u_{x}}{\partial x^{2}}+\frac{\partial^{2} u_{y}}{\partial y^{2}}+\frac{\partial^{2} u_{z}}{\partial z^{2}}\right)+\left(\frac{\partial^{2} u_{x}}{\partial x^{2}}+\frac{\partial^{2} u_{y}}{\partial y^{2}}\right)+\frac{\partial^{2} u_{z}}{\partial z^{2}}\right)\right]-\frac{2 \mu}{3 \varepsilon_{p}}\left(\frac{\partial^{2} u_{x}}{\partial x^{2}}+\frac{\partial^{2} u_{y}}{\partial y^{2}}+\frac{\partial^{2} u_{z}}{\partial z^{2}}\right) l-\left(\frac{\mu}{\kappa_{b r}}+\beta_{F}|u|+Q_{b r}\right) u+F_{z}$

$\nabla \cdot\left[-\frac{\eta}{\varepsilon_{p}}\left(\left(\frac{\partial^{2} u_{x}}{\partial x^{2}}+\frac{\partial^{2} u_{y}}{\partial y^{2}}+\frac{\partial^{2} u_{z}}{\partial z^{2}}\right)+\left(\frac{\partial^{2} u_{x}}{\partial x^{2}}+\frac{\partial^{2} u_{y}}{\partial y^{2}}+\frac{\partial^{2} u_{z}}{\partial z^{2}}\right)^{T}+p I\right)=-\frac{\eta}{k} u\right.$

Brinkman equation in axial direction

$\frac{\rho}{\varepsilon_{p}}\left(\frac{\partial u_{x}}{\partial t}+u_{x} \frac{\partial u_{x}}{\partial x}+u_{y} \frac{\partial u_{x}}{\partial y}+u_{z} \frac{\partial u_{z}}{\partial z}\right)=\left[-\frac{\partial p}{\partial x}+\frac{\mu}{\varepsilon_{p}}\left(\left(\frac{\partial^{2} u_{y}}{\partial x^{2}}+\frac{\partial^{2} u_{y}}{\partial y^{2}}+\frac{\partial^{2} u_{y}}{\partial z^{2}}\right)+\left(\frac{\partial^{2} u_{y}}{\partial x^{2}}+\frac{\partial^{2} u_{y}}{\partial y^{2}} \frac{\partial^{2} u_{y}}{\partial z^{2}}\right)^{T}\right)\right]-\frac{2 \mu}{3 \varepsilon_{p}}\left(\frac{\partial^{2} u_{y}}{\partial x^{2}}+\frac{\partial^{2} u_{y}}{\partial y^{2}}+\frac{\partial^{2} u_{y}}{\partial z^{2}}\right) l-\left(\frac{\mu}{\kappa_{b r}}+\beta_{F}|u|+Q_{b r}\right) u+F_{z}$

$\nabla \cdot\left[-\frac{\eta}{\varepsilon_{p}}\left(\left(\frac{\partial^{2} u_{y}}{\partial x^{2}}+\frac{\partial^{2} u_{y}}{\partial y^{2}}+\frac{\partial^{2} u_{y}}{\partial z^{2}}\right)+\left(\frac{\partial^{2} u_{y}}{\partial x^{2}}+\frac{\partial^{2} u_{y}}{\partial y^{2}}+\frac{\partial^{2} u_{y}}{\partial z^{2}}\right)^{T}+p I\right)=-\frac{\eta}{k} u\right.$

Brinkman equation in tangential direction

$\frac{\rho}{\varepsilon_{p}}\left(\frac{\partial u_{z}}{\partial t}+u_{x} \frac{\partial u_{z}}{\partial x}+u_{y} \frac{\partial u_{z}}{\partial y}+u_{z} \frac{\partial u_{z}}{\partial z}\right)=\left[-\frac{\partial p}{\partial z}+\frac{\mu}{\varepsilon_{p}}\left(\left(\frac{\partial^{2} u_{z}}{\partial x^{2}}+\frac{\partial^{2} u_{z}}{\partial y^{2}}+\frac{\partial^{2} u_{z}}{\partial z^{2}}\right)+\left(\frac{\partial^{2} u_{z}}{\partial x^{2}}+\frac{\partial^{2} u_{z}}{\partial y^{2}} \frac{\partial^{2} u_{z}}{\partial z^{2}}\right)^{T}\right)\right]-\frac{2 \mu}{3 \varepsilon_{p}}\left(\frac{\partial^{2} u_{z}}{\partial x^{2}}+\frac{\partial^{2} u_{z}}{\partial y^{2}}+\frac{\partial^{2} u_{z}}{\partial z^{2}}\right) l-\left(\frac{\mu}{\kappa_{b r}}+\beta_{F}|u|+Q_{b r}\right) u+F_{z}$

$\nabla \cdot\left[-\frac{\eta}{\varepsilon_{p}}\left(\left(\frac{\partial^{2} u_{z}}{\partial x^{2}}+\frac{\partial^{2} u_{z}}{\partial y^{2}}+\frac{\partial^{2} u_{z}}{\partial z^{2}}\right)+\left(\frac{\partial^{2} u_{z}}{\partial x^{2}}+\frac{\partial^{2} u_{z}}{\partial y^{2}}+\frac{\partial^{2} u_{z}}{\partial z^{2}}\right)^{T}+p I\right)=-\frac{\eta}{k} u\right.$

Mass transfer

$\frac{\partial c_{i}}{\partial r}+\nabla \cdot\left(-D_{i}\left(\frac{1}{r} \frac{\partial}{\partial r}\left(r c_{i r}\right)+\frac{1}{r} \frac{\partial c_{i \theta}}{\partial \theta}+\frac{\partial c_{i z}}{\partial z}\right)\right)+u \cdot\left(\left(\frac{1}{r} \frac{\partial}{\partial r}\left(r c_{i r}\right)+\frac{1}{r} \frac{\partial c_{i \theta}}{\partial \theta}+\frac{\partial c_{i z}}{\partial z}\right)\right)=R_{i}$

$N_{i}=-D_{i}\left(\frac{1}{r} \frac{\partial}{\partial r}\left(r c_{i r}\right)+\frac{1}{r} \frac{\partial c_{i \theta}}{\partial \theta}+\frac{\partial c_{i z}}{\partial z}\right)+u \cdot c_{i}$

$\nabla\left[\rho \omega_{i} u-\rho \omega_{i} \sum_{j=1}^{n} \tilde{D}_{i j}\left(\nabla x_{j}+\left(x_{j}-\omega_{j}\right) \frac{\nabla p}{p}\right)-D_{i}^{T} \frac{\nabla T}{T}\right]=R_{i}$ 
The equation below is derived from the Gauss divergence theorem.

$$
\begin{aligned}
\int_{\Omega} & \rho \frac{\partial c}{\partial t} \vec{u}^{*} d \Omega+\int_{\Omega}\left\{(D \nabla c \cdot \nabla u+\beta c u+u \cdot \nabla c) \vec{u}^{*}\right\} d \Omega \\
& -\int_{\Gamma} u A \nabla c \cdot n d \Gamma \\
= & \int_{\Omega} R \vec{u}^{*} d \Omega
\end{aligned}
$$

Finally, by substitution of the boundary conditions, the following form will be generated:

$$
\begin{aligned}
\int_{\Omega} & \rho \frac{\partial c}{\partial t} \vec{u}^{*} d \Omega+\int_{\Omega}\left\{\left(D \nabla c \cdot \nabla \vec{u}^{*}\right)+u \cdot \nabla c \vec{u}^{*}+\beta c \vec{u}^{*}\right\} d \Omega \\
& +\int_{\Gamma_{3}} \sigma c \vec{u}^{*} d \Gamma \\
= & \int_{\Omega} R \vec{u}^{*} d \Omega+\int_{\Gamma_{2}} g_{2} \vec{u}^{*} d \Gamma+\int_{\Gamma_{3}} g_{3} \vec{u}^{*} d \Gamma
\end{aligned}
$$

where $c(x, t)$ with $t=0, c_{\Gamma_{1}}=g_{1}$, beside $g_{2}$ and $g_{3}$ are the boundary conditions.

The solution $c$ is approximated by a linear combination of the basis time-independent basis functions.

$c_{h}(x, t)=\sum_{j=1}^{n} c_{j}(t) \varphi_{j}(x)+c_{0}(x, t)$

For the test function $v(x)$ again the basis functions $\varphi_{i}(x)(i=1$, $2, \ldots, n)$ are substituted. Finally we arrive at the Galerkin formulation:

$$
\begin{aligned}
\sum_{j=1}^{n} & \frac{\partial c_{j}}{\partial t} \int_{\Omega} \varphi_{i} \varphi_{j} d \Omega \\
& +\sum_{j=1}^{n} c_{j}\left\{\begin{array}{c}
\int_{\Omega}\left[\left(D \nabla \varphi_{j} \cdot \nabla \varphi_{i}\right)+\left(u \cdot \nabla \varphi_{j}\right) \varphi_{i}\right. \\
\left.+\beta \varphi_{i} \varphi_{j}\right] d \Omega \\
+\int_{\Gamma_{3}} \sigma \varphi_{i} \varphi_{j} d \Gamma
\end{array}\right\} \\
= & \int_{\Omega} R \varphi_{i} d \Omega \int_{\Gamma_{2}} g_{2} \varphi_{i} d \Gamma+\int_{\Gamma_{3}} g_{3} \varphi_{i} d \Gamma-\int_{\Omega}\left\{\left(D \nabla c_{0} \cdot \varphi_{i}\right)\right. \\
& \left.+\beta c_{0} \varphi_{i}\right\} d \Omega-\int_{\Omega} \frac{\partial c_{0}}{\partial t} \varphi_{i} d \Omega i \\
= & 1(1) n
\end{aligned}
$$

It is clear that this equation generates $n$ unknown parameters within $n$ linear ordinary differential equations, which can be summarized in matrix-vector of:

$M \dot{c}+S c=F$

where $M$ defines the mass matrix, $S$ is the so-called stiffness matrix and - defines differentiation with respect to time. The elements of the matrices and right-hand side are defined by:

$$
\begin{aligned}
m_{i j}= & \int_{\Omega} \varphi_{i} \varphi_{j} d \Omega \\
s_{i j}= & \int_{\Omega}\left\{\left(D \nabla \varphi_{j} \cdot \varphi_{j}\right)+\left(u \cdot \nabla \varphi_{j}\right) \varphi_{j}+\beta \varphi_{i} \varphi_{j}\right\} d \Omega \\
& +\int_{\Gamma_{3}} \sigma \varphi_{i} \varphi_{j} d \Gamma \\
F_{i}= & \int_{\Omega} R \varphi_{i} d \Omega-\int_{\Omega}\left\{\left(D \nabla c_{0} \cdot \nabla \varphi_{i}\right)+\left(u \cdot \nabla c_{0}\right) \varphi_{i}+\beta c_{0} \varphi_{i}\right\} d \Omega \\
& -\int_{\Omega} \frac{\partial c_{0}}{\partial t} \varphi d \Omega+\int_{\Gamma_{2}} g_{2} \varphi_{i} d \Gamma+\int_{\Gamma_{3}} g_{3} \varphi_{i} d \Gamma
\end{aligned}
$$

The only difference of this matrix with the potential problem is the extra convective terms in the stiffness matrix and the extra parts of time-derivative with the mass matrix. Hence, the stiffness matrix becomes non-symmetric by means of extra convective terms. Generally, the mass-matrix can be computed by a quadrature rule. In general $M$ and $S$ have similar structures.

\section{Numerical solution of equations}

In porous domains, the control volume must be large enough to contain solid matrix elements with its pores, but it also must be small in contrast with the typical macroscopic dimensions of the problem. Thus, $1.72 \mathrm{M}$ elements were considered as the conformal mesh employs a symmetry boundary condition along the device's vertical mid-surface.

Combining the equations and solving them was done by PARDISO analyzer. It is a memory efficient and high performance usage to solve large sparse linear systems of equations by shared multiprocessors. The PARDISO calculates the solution of a set of sparse linear equations in a regular setting of 3 different $n$ by $n$ matrices $(A \times X=B)$. The analysis steps were performed by a parallel $L U, L D L$ or $L L^{T}$ factorization. Generally, there are two types of analyzing based on the input matrix; either symmetric or unsymmetric. In this research, as mentioned before, the input matrix is symmetric. The symmetric fill-in reducing permutation $P$ is computed based on the either the nested dissection algorithm from the METIS package or the minimum degree algorithm. The solver first computes a symmetric fill-in reducing permutation $P$ based on either the minimum degree algorithm or the nested dissection algorithm along with the METIS algorithm for computing partitionings and fill-reducing orderings. In this algorithm, the parallel left-right looking numerical Cholesky factorization was used (by means of $P A P^{T}=L D L^{T}$ for symmetric indefinite matrices, or $P A P^{T}=L L^{T}$ for symmetric positive-definite matrices). In the next step, $1 \times 1$ and $2 \times 2$ Bunch and Kaufman pivoting or diagonal pivoting is used to symmetric indefinite matrices; besides, forward and backward substitutions are also used for approximation of $X$ and refining the iterative.

In this work COMSOL Multiphysics (Version 4.2.a, 2011) software was used at the Department of Chemical Engineering, University of Malaya. In this software, UMFPACK solver was utilized for error control $[20,21]$ and finite element method (FEM) was used for the numerical solutions of equations [22]. The complete mesh consisted of $2 \times 10^{5}$ tetrahedral elements. The computational time to solve the set of equations was about 5 days using a PC of Intel ${ }^{\circledR}$ Core $^{\mathrm{TM}} 2$ Duo CPU and RAM of $8 \mathrm{~GB}$.

\section{Results and discussion}

\subsection{Hydrodynamic simulation}

The numerical solution presented in the previous sections allowed us to determine the fluid flow velocity, Reynolds number, concentration gradients, convective and diffusive fluxes, reaction yield as well as concentrations of each component in ceramic membrane and catalytic bed in each cell.

Three-dimensional velocity vectors within the catalytic bed and ceramic membrane are shown in Fig. 4. Both cross-section images were obtained from the middle of the system. The vectors indicate a highly superficial flow direction in comparison with the radial ones. Because the porosity of the ceramic membrane in comparison with the catalytic bed is much lower. It is worth mentioning that the highest velocity was observed at the center of the catalytic bed, but this point came down gently by getting closer to the end of the catalytic bed which is due to gravitational force. 


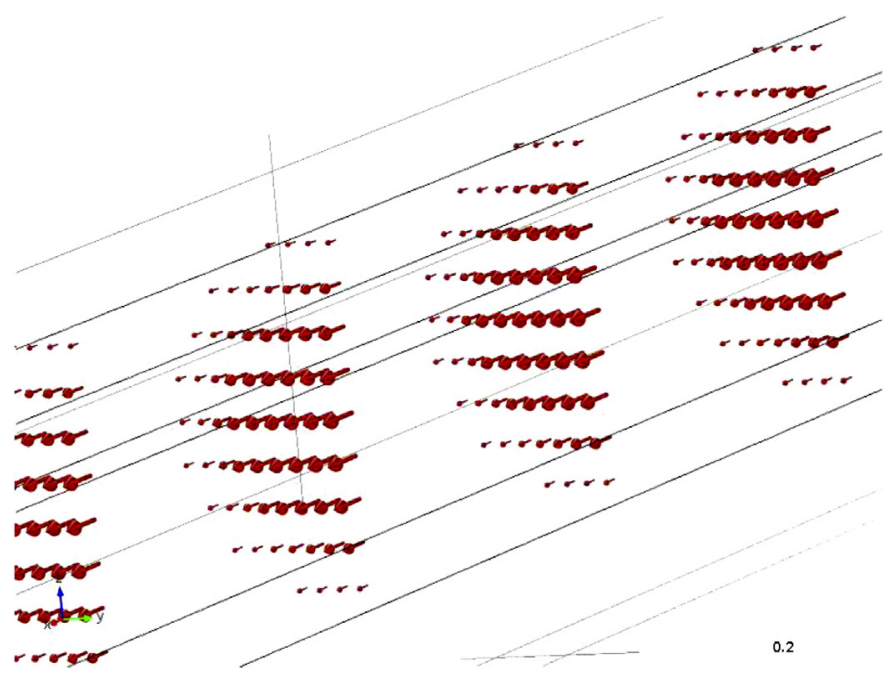

(a)

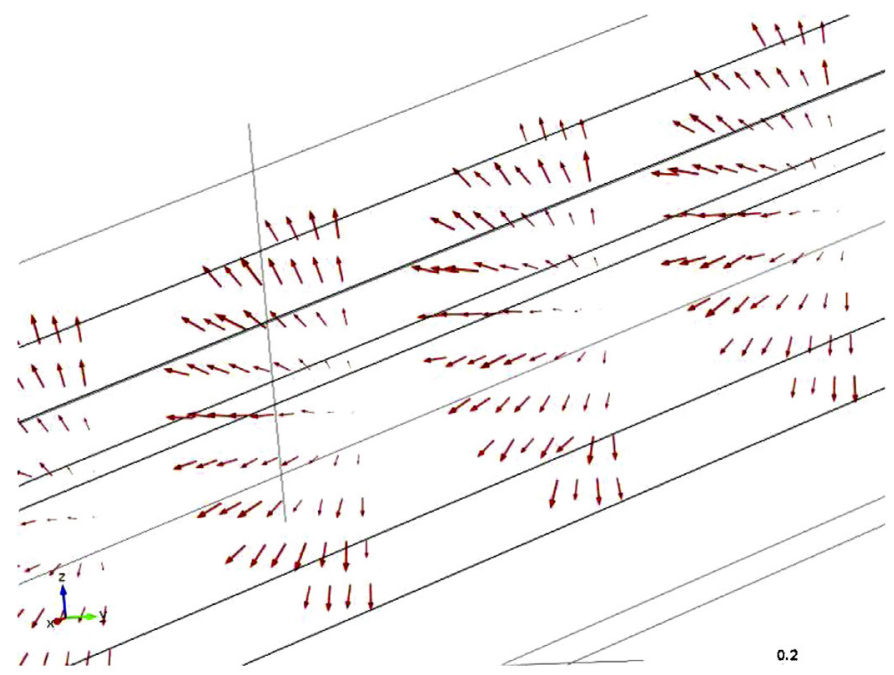

(b)

Fig. 4. Velocity vectors within the catalytic bed and ceramic membrane at steady state condition (after $120 \mathrm{~s}$ ): (a) $x$-direction velocity and (b) resultant of $y$ and $z$ direction velocity.

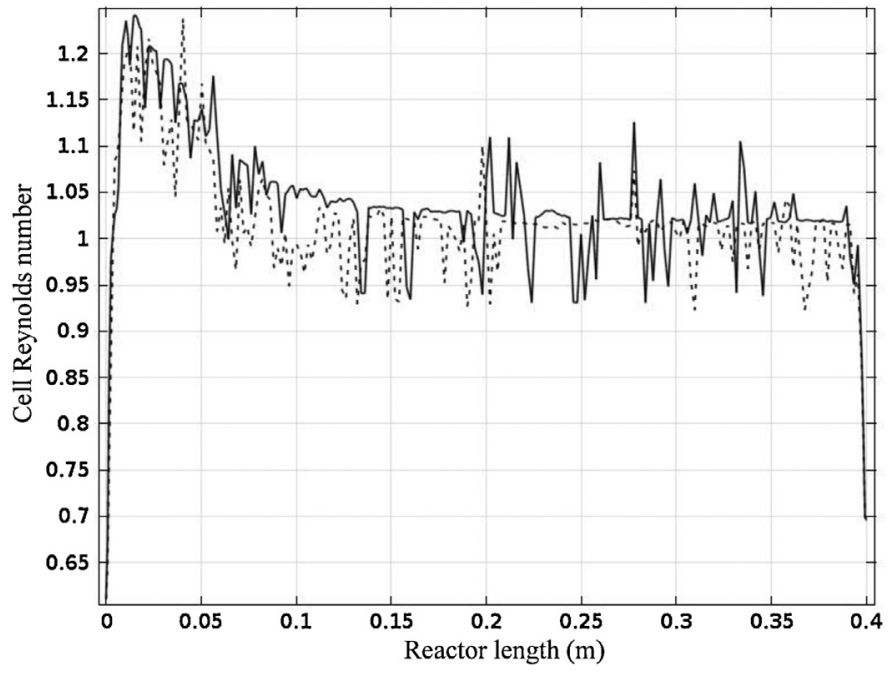

Fig. 5. Cell Reynolds number at steady state situation: ..., upper interface between catalytic bed and solid membrane; -, downer interface between catalytic bed and solid membrane.
In addition, the magnitudes of the velocity vectors through the mentioned system in different times and at steady state situation are shown in Fig. 5.

As the flow entered into the catalytic bed, it passed through the ceramic membrane as well. The simulation results show that the material had $85 \mathrm{~s}$ residence time.

The cell Reynolds number at the upper and downer interfaces between the catalytic bed and the solid membrane at steady state situation has been discussed in Fig. 5. Generally, this graph shows that Reynolds number is very low in the system, which causes the numerical solution converges sooner; because convergence was achieved at less iteration. In addition, Reynolds number decreased in the flow direction at the first section of the reactor and then became stable. These changes depend on the flow pattern and molar concentration of different components. However the effect of component concentration was less, because at the starting point both TG concentration (with higher density and viscosity) and methanol concentration (with less density and viscosity) were high. The concentration of the production increased in the middle and end sections of the membrane reactor, which has less density or viscosity in comparison with triglyceride. But the concentration of methanol decreased. It was observed that the components negated the effect of each other. Another point is the amount of the Reynolds number, which is fewer at the upper interface, maybe because of the gravity force.

The local velocity magnitude at $60{ }^{\circ} \mathrm{C}$ for the ceramic membrane and the catalytic bed can be seen in Fig. 7. As we see from the figure, it took almost $85 \mathrm{~s}$ for the material to reach the end of the catalytic bed and become steady. In this period, diffusion of the components in the ceramic membrane needed more time (nearly $300 \mathrm{~s}$ ). This period is also matched with the velocity difference between the catalytic bed and the ceramic membrane, which is almost 2.5 times. Then we selected $400 \mathrm{~s}$ for a complete steady state situation in both membrane and catalytic bed. Comparing the experimental results with the CFD simulation, we found that CFD simulation had a deviation of $8 \%$ at the outlet section of the catalytic bed for both of the $50\left(0.0179 \times 10^{-3} \mathrm{~m}\right)$ and 60 $\left(0.0195 \times 10^{-3} \mathrm{~m}\right){ }^{\circ} \mathrm{C}$. Regarding to find a suitable time of computational running which was unknown from experimental part, the component concentration of two different surfaces were controlled, the first one at the catalytic bed output and the other one at the outside of ceramic surface. Then, different times were applied. The highest time by which material reached both of the

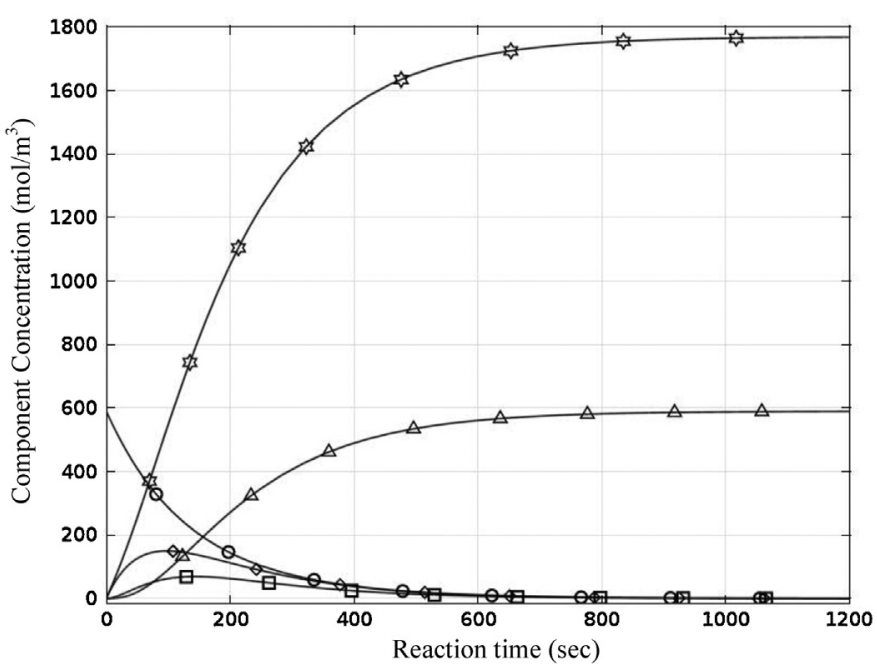

Fig. 6. Concentration distribution $\left(\mathrm{mol} / \mathrm{m}^{3}\right)$ within the catalytic bed at different times. 放, Fatty acid methyl ester; $\Delta$, glycerol; $\bigcirc$, triglyceride; $\diamond$, diglyceride; $\square$, monoglyceride. At $60^{\circ} \mathrm{C}$. 
surfaces and concentration became stable was selected as the steady state time.

\subsection{Convection and diffusion}

As mentioned before three reversible reactions with different rate constants $\left(k_{n}\right)$ should be done for biodiesel production (Table 3). A molecule of fatty acid methyl ester was produced at each of these three stages, and three molecules of alcohol were required to produce three molecules of ester and one molecule of glycerol. In this work, methanol was used as the alcohol molecule with the volume fraction of 1:1 compared with the triglyceride at the temperature of $60^{\circ} \mathrm{C}$. The simulation result is shown in Fig. 6 .

The comparison of the CFD simulation with the experimental results shows $0.57 \%$ overestimations of CFD results. One possible explanation for this is the importance of reversible reactions. Since Darnoko and Cheryan [16] provided data only for the forward reactions $\left(\mathbf{k}_{1}, \mathbf{k}_{2}\right.$, and $\mathbf{k}_{3}$ ), so in this work the reversible reactions were neglected. From the comparison of the concentration curves, the highest production rates of FAME, DG and MG were observed between the 1st and the 3rd minute of the process. Then FAME production rate decreased until the equilibrium point; and at the same time the concentration of MG and DG increased till the peak and after that decreased very slowly. The increase in FAME concentration was followed by an increase in $G$ concentration, which both of them released from TG molecules. But, due to the production of intermediate components such as DG and MG, the relative proportion of $G$ was not always the same as FAME.

The most important aim of this work is to investigate and compare the role of convection and diffusion.

In Fig. 8(a-f), the magnitude of convective and diffusive flux of all components is shown at the steady state situation and the temperature of $60{ }^{\circ} \mathrm{C}$. The first picture belongs to triglyceride that shows TG cannot pass through the ceramic membrane (in both of the convection and diffusion terms). Triglyceride (TG) was not miscible in $\mathrm{MeOH}$. TG is appeared in form of small droplets during the transesterification reaction. Therefore, the produced biodiesel which consists of fatty acid alkyl esters with small molecular sizes was able to pass through the membrane along with alcohol and glycerol while the large droplet of oil cannot pass through the
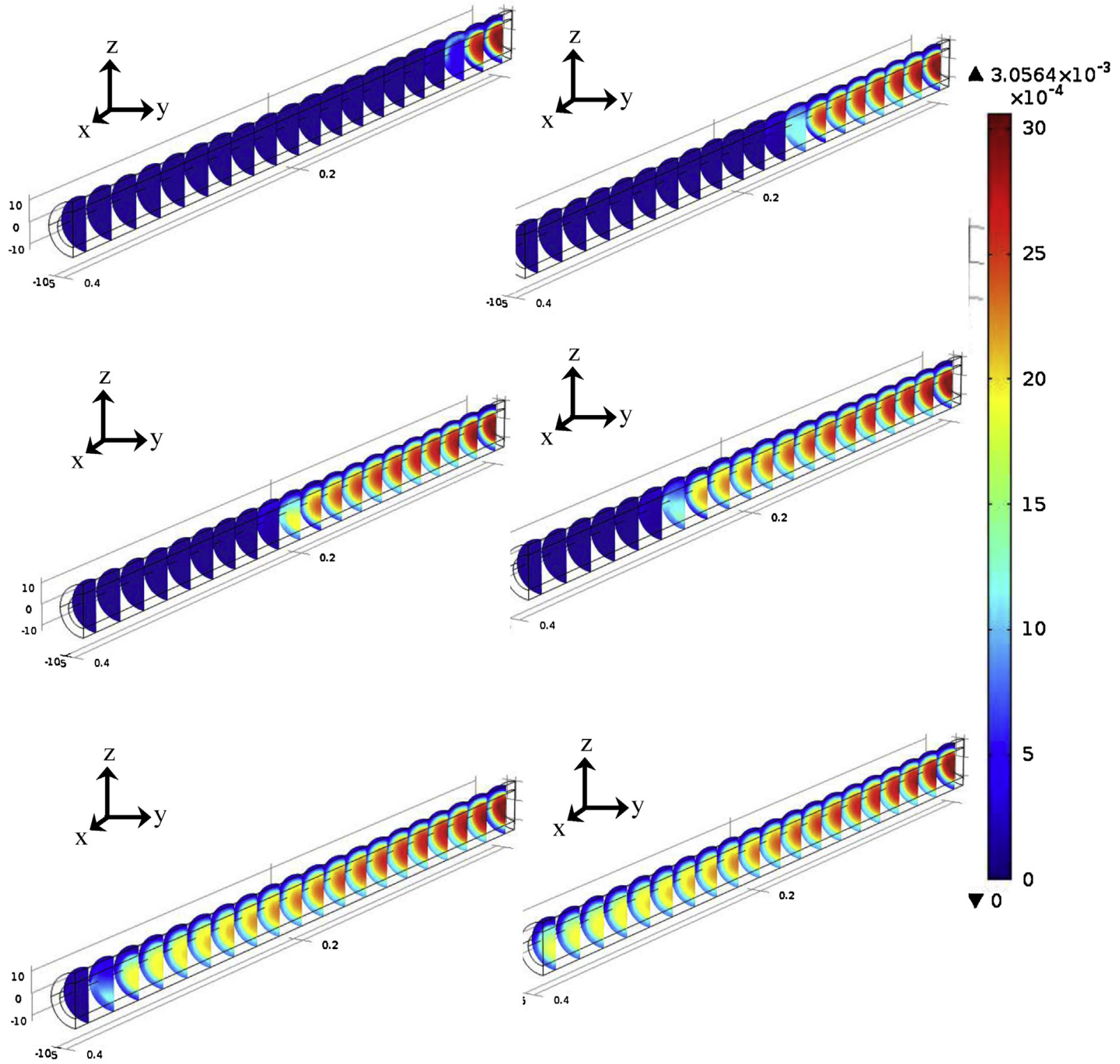

Fig. 7. Velocity magnitude $(\mathrm{m} / \mathrm{s})$ within the catalytic bed and ceramic membrane. 

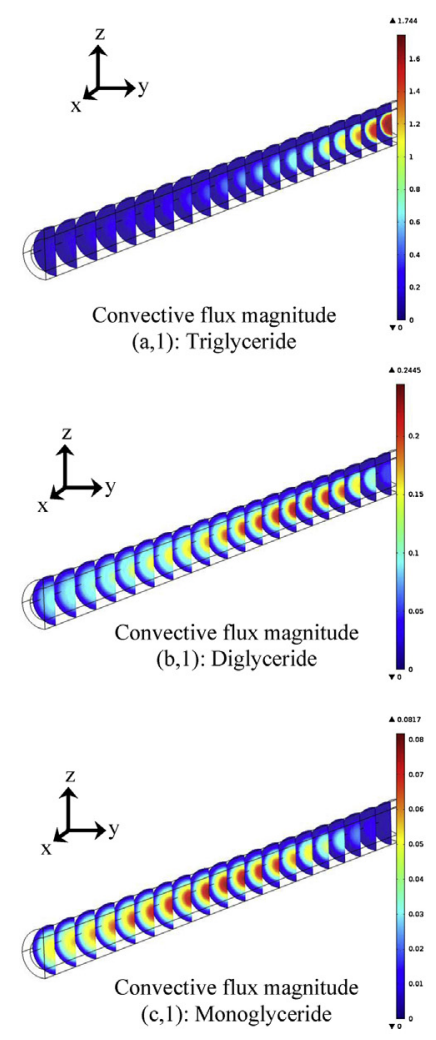

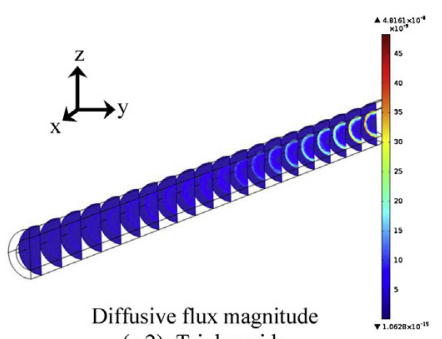
(a,2): Triglyceride
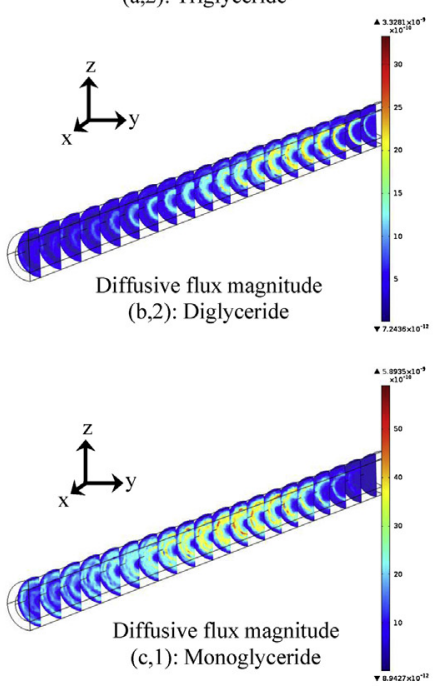
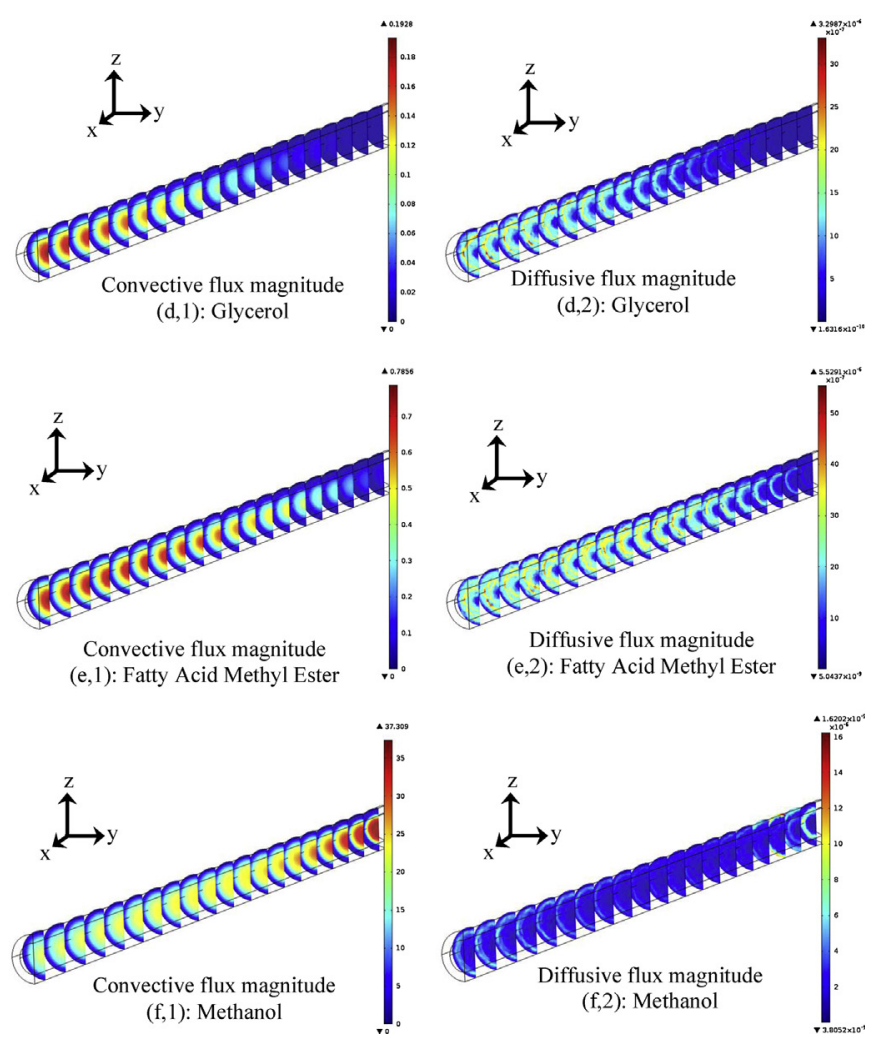

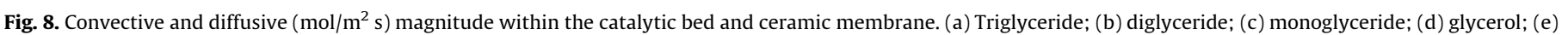
fatty acid methyl ester; (f) methanol.

membrane pores. Several important points can observe at this figure. First, at highest fluid velocity $\left(30 \times 10^{-4} \mathrm{~m} / \mathrm{s}\right)$, the magnitude ratio between convection and diffusion is about $3.6 \times 10^{7}$. After that, the convection sharply decreases to the value of $0.4 \mathrm{~mol} / \mathrm{m}^{2} \mathrm{~s}$ at the center of catalytic bed and then it slightly decreases to reach the value of $0.2 \mathrm{~mol} / \mathrm{m}^{2} \mathrm{~s}$ at the end of the catalytic bed. According to Brinkman equation (Eq. (7)), this decreasing rate is caused by two factors. Firstly, the reduction of fluid flow velocity (from $30 \times 10^{-4} \mathrm{~m} / \mathrm{s}$ at the inlet to $19 \times 10^{-4} \mathrm{~m} / \mathrm{s}$ at the outlet) and secondly reduction of the TG concentration (from 600 to $70 \mathrm{~mol} / \mathrm{m}^{3}$ ).

The next part of this Fig. 8(a2) shows by increasing the radial distance from the center of the catalytic bed, the value of the TG diffusion is increasing while the convection is decreasing. It can be justified that, by increasing the radial distance, the average of fluid velocity is decreasing. According to Fick's law the mass transfer caused by diffusion at the surface of components is described by the equation below,

$N_{A}=-\left.D_{A B} \frac{d\left(C_{A}-C_{A S}\right)}{d u}\right|_{y=0}$

where the $\left(C_{A}-C_{A S}\right)$ is the component concentration difference between two boundaries. Therefore diffusion comes to zero, when the concentrations become similar. By decreasing the fluid velocity, more time will be available for diffusion to achieve the steady state situation. Thus the diffusion is more prominent beside the ceramic membrane where the concentrations can reach to the equal values.

However the increasing rate of diffusion is more prominent at the length of $20 \mathrm{~cm}$ from the reactor inlet. Furthermore the final value for both convection and diffusion (at catalytic bed output) shows the reaction has not been completed even at the end of reactor.
The second and third graphs indicate the magnitude of convection and diffusion of DG and MG, respectively. The highest convection value of $0.2445 \mathrm{~mol} / \mathrm{m}^{2} \mathrm{~s}$ is observed for DG within the first $17 \mathrm{~cm}$ length of the catalytic bed from the inlet part. Generally, the values are more prominent from 5 to $17 \mathrm{~cm}$ of the catalytic bed which depends on its increasing concentration due to the related reaction. At this section, the diffusion magnitude also reached to the value of $23-30 \mathrm{~mol} / \mathrm{m}^{2} \mathrm{~s}$. The convective and diffusive flow pattern of MG is also similar to the DG. However, since DG was produced by the first reaction and MG by the second one, so DG reached the peak of its convective flux sooner, exactly alike its concentration graph in Fig. 6. Therefore, the highest value of MG convection of $0.08 \mathrm{~mol} / \mathrm{m}^{2} \mathrm{~s}$ and diffusion value of $4 \times 10^{-9}$ $5 \times 10^{-9} \mathrm{~mol} / \mathrm{m}^{2} \mathrm{~s}$ are observed within the $13-26 \mathrm{~cm}$ of the catalytic bed from the inlet section. Similar to TG, these are not consumed completely and a percentage of them remained in the outlet production, which is more important in MG concentration. Therefore the values of $0.05 \mathrm{~mol} / \mathrm{m}^{2} \mathrm{~s}$ and $2.2 \times 10^{-9} \mathrm{~mol} / \mathrm{m}^{2} \mathrm{~s}$ are still observed at the outlet for both convection and diffusion respectively. Another worth noting point is the role of diffusion in MG which is more highlighted than DG because of its smaller molecular size. For both of these components the role of diffusion at the center of the catalytic bed was nearly negligible. It is worth mentioning that by decreasing the concentration of these two components, their diffusion decreases in the catalytic bed as well. But since no reaction was assumed in the ceramic membrane, the amount of DG and $G$ which entered into the membrane remained stable without any changing.

Glycerol is the third important component which its convection and diffusion parameters are increased from $16 \mathrm{~cm}$ from the inlet section to its outlet. Therefore the highest convection and diffusion of $0.1928 \mathrm{~mol} / \mathrm{m}^{2} \mathrm{~s}$ and $2.2 \times 10^{-6} \mathrm{~mol} / \mathrm{m}^{2} \mathrm{~s}$ are observed at the outlet sections. Similar to the other components, the diffusion is 
more prominent at the membrane section and the places were the fluid velocity decreased and the diffusion is negligible especially at the center of the reactor where convective magnitude is more important.

The patterns of convective and diffusive flux of $G$ and FAME are quite similar however the role of convection and diffusion of FAME is more prominent. Thus, the highest convection magnitude of 0.7856 and diffusive magnitude of $5.529 \times 10^{-6} \mathrm{~mol} / \mathrm{m}^{2} \mathrm{~s}$ are observed at the outlet of reactor which are much more than glycerol. Furthermore, both convection and diffusion of FAME sharply increase by starting the first reaction.

Generally the ratio of convective to diffusive magnitudes is decreasing within the range of $7.3 \times 10^{7}, 1.38 \times 10^{7}, 5.8 \times 10^{5}$ and $2.6 \times 10^{5} \mathrm{~mol} / \mathrm{m}^{2} \mathrm{~s}$ for DG, MG, G and FAME, respectively.

The last figure belongs to the methanol. We can see that the magnitude of convective flux decreased by reducing the methanol concentration and velocity within the catalytic bed from 37.309 to $22.7 \mathrm{~mol} / \mathrm{m}^{2} \mathrm{~s}$. However, the magnitude of diffusion is completely different; at first, its diffusion increases to about $7 \times 10^{-5} \mathrm{~mol} / \mathrm{m}^{2} \mathrm{~s}$ due to its high concentration and then it sharply decreased to nearly zero (at the middle of the reactor) due to the role of the other reactions which result in increasing the concentration of the other components. Finally, diffusion increases again because of its extra concentration. There are more details in Fig. 9(d) which will be explained in the next section.

Fig. 9 shows the same result of Fig. 8 but in different times till the steady state conditions. We selected 6 different times $(50,100$, $150,200,300$ and $400 \mathrm{~s}$ ).

It is worth mentioning that in all graphs the decreasing section which reached zero value has not been investigated. The only reason is the material which did not approach to the end of reactor and also the concentration was very low in these sections.

Fig. 9(a and b) describes the convection and diffusion of DG and MG at different times. It is observed that both DG and MG followed the similar pattern as mentioned before, but two differences are observed at the diagrams.

Firstly, due to the higher reaction rate of second reaction which results in production of DG, the highest concentration of DG (consequently followed by its maximum convection of $0.09 \mathrm{~mol} /$ $\mathrm{m}^{2} \mathrm{~s}$ ) is started after $200 \mathrm{~s}$ at the middle of reactor exactly. For MG its maximum convection of $0.04 \mathrm{~mol} / \mathrm{m}^{2} \mathrm{~s}$ is started after $300 \mathrm{~s}$ at the $30 \mathrm{~cm}$ from the reactor inlet. Meanwhile, the highest diffusion value for DG and MG are observed after 50 and 150 s respectively, from the starting time of reaction. It is also observed that the diffusion of DG decreases after its highest value to the value of $11 \mathrm{~mol} / \mathrm{m}^{2} \mathrm{~s}$ at the time of $200 \mathrm{~s}$. The reason is due to the consumption of these components at the next reaction. Finally the value of DG diffusion became stable at the value of $16 \mathrm{~mol} / \mathrm{m}^{2} \mathrm{~s}$. This section is exactly matched with decreasing rate of convective magnitude. On the other hand, increasing rate of diffusion is due to decreasing the convection. However, at the steady state condition, both of the convection and diffusion, which are dependent on the component concentration, decreased. This pattern is repeated for MG as well. However, the decreasing rate of diffusion is started after $150 \mathrm{~s}$ from the highest value of $50 \mathrm{~mol} / \mathrm{m}^{2} \mathrm{~s}$ to the value of $45 \mathrm{~mol} / \mathrm{m}^{2} \mathrm{~s}$. After this time the diffusion of MG became stable at the maximum value of $30 \mathrm{~mol} / \mathrm{m}^{2} \mathrm{~s}$.

In addition the highest convection magnitude of DG is more than twice of the highest convection of MG ( $9 \mathrm{~mol} / \mathrm{m}^{2} \mathrm{~s}$ compared to the $4 \mathrm{~mol} / \mathrm{m}^{2} \mathrm{~s}$, respectively). Meanwhile the highest diffusion
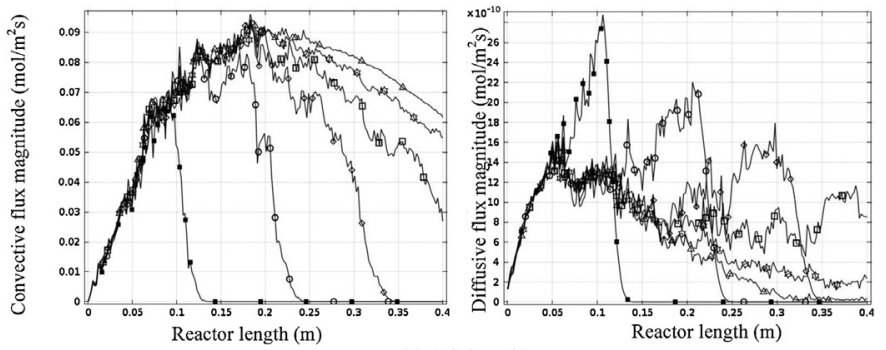

(a), Diglyceride

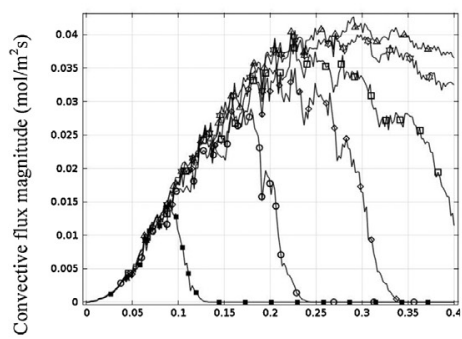

Reactor length $(\mathrm{m})$

(b), Monoglyceride

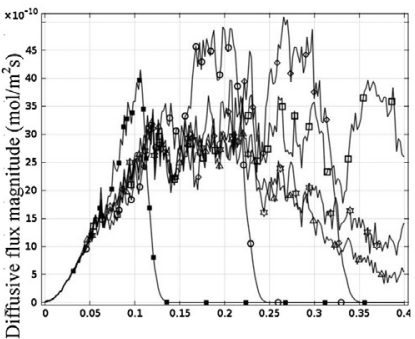

Reactor length (m)
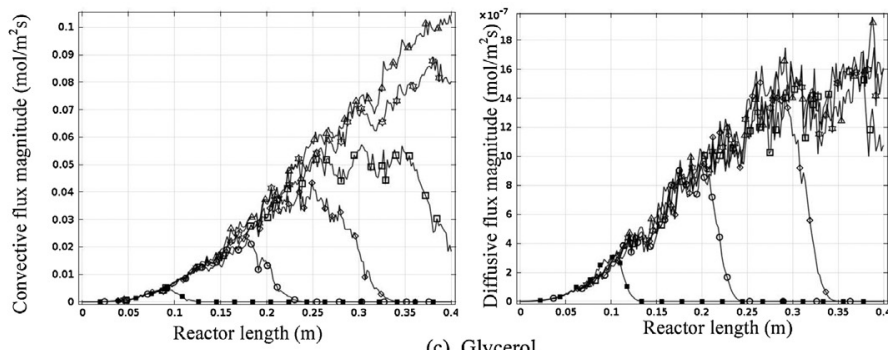

(c), Glycerol
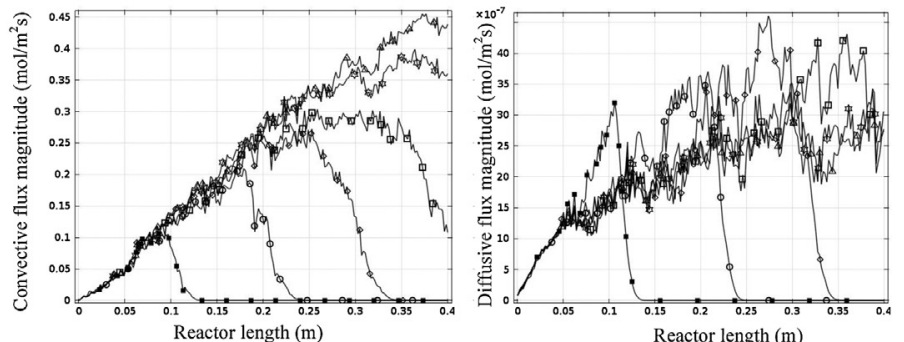

(d), Fatty Acid Methyl Ester
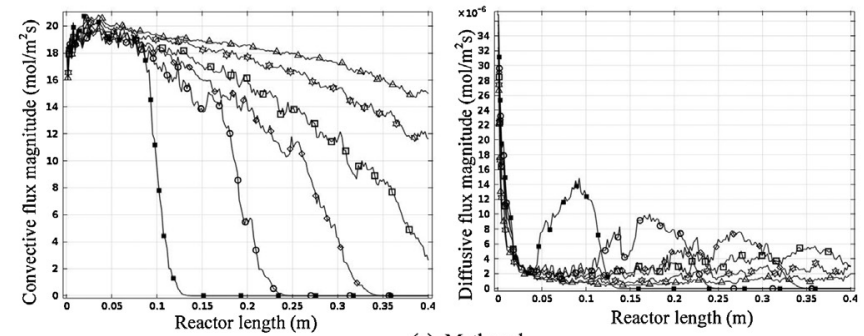

(e), Methanol

Fig. 9. Concentration distribution of different components $\left(\mathrm{mol} / \mathrm{m}^{3}\right)$ at the ceramic membrane. monoglyceride; (c) glycerol; (d) fatty acid methyl ester; (e) methanol. 
of the MG is nearly twice of the highest value of DG diffusion value. The reason depends on their difference in molecular structure which directly affects the diffusion coefficient.

For $\mathrm{G}$ and FAME, the situation is different, because both of these materials were produced but not consumed, so both of their convective and diffusive flux increased by time. As mentioned before, the increasing rate of FAME was three times bigger than G, then the convective and diffusive magnitude of FAME was also more than G. Despite of DG and MG, G and FAME do not have a maximum value because of the reactions regularity. The diagrams show even after $400 \mathrm{~s}$, the convection and diffusion of FAME and G did not achieve to the stable value which is another reason that the reactor length was not enough to complete the reaction.

Finally, the last figure is about the $\mathrm{MeOH}$. As the concentration is not zero at the first step, both of the convective and diffusive flux magnitudes were started from a high value. The convective flux peaked at $0.025 \mathrm{~m}$ of the reactor but diffusive value decreased till the minimum value of $1 \mathrm{~mol} / \mathrm{m}^{2} \mathrm{~s}$ and this is because of the high velocity at the entrance of the reactor. After that, the convective magnitude decreased in spite of the diffusive value which became stable. It depends on the existence of the other components (produced by two important reactions and result in DG and MG production) at the middle section of the reactor, which can affect the methanol diffusion. By consumption of DG and MG, the diffusion rose again but slowly because of the existence of $G$ and MES. Because of the reduction in fluid flow velocity, the convection in the process decreased from $0.05 \mathrm{~m}$ of the reactor entrance. Generally, the rate of methanol convection is decreased at different times, which is due to decreasing of its concentration.

\section{Conclusion}

In this paper, a ceramic membrane packed with catalytic bed was employed for production of high quality biodiesel. $\mathrm{TiO}_{2} / \mathrm{Al}_{2} \mathrm{O}_{3}$ was used as a micro porous ceramic membrane and the potassium hydroxide supported on palm shell activated carbon was used as the catalyst. Thus, the system was a combination of the reaction and separation inside one single shell. Most of the CFD studies in membrane devices were investigated on some parameters such as pressure distribution and velocity fields or component concentrations at steady-state conditions. However, convection and diffusion phenomena are of paramount importance. This work is aimed to use 3D CFD simulation to investigate hydrodynamic parameters, Reynolds number and compute convection as well as diffusion mechanisms at unsteady state situations. The continuity, NavierStokes, Brinkman and Stephan-Maxwell were the equations employed to simulate the system and were solved by the PARDISO algorithm which supports parallel processes.

From hydrodynamic parameters, it was found that the fully developed assumption in membrane flows is applicable since the $R e$ number in such systems is very low and limited in laminar range. Investigation of velocity is the best suggestion for finding the optimum value of the components' residence time and reactor length. In our case we found that the residence time is not matched with the reactor length and it affected the reaction efficiency [23]. From convection and diffusion parameters, it was found that at the area near and within the ceramic membrane wall, the diffusion term is more prominent while at the middle section, the convection term is more applicable for all components. But an important point is the role of diffusion in MG which is more highlighted than DG. It depends on the molecular size of MG which is smaller than DG. Thus it can enter into the ceramic membrane easier, especially by diffusion. For both of these components the role of diffusion at the center of catalytic bed is nearly negligible. The pattern of convective and diffusive flux of $G$ and FAME is also similar. The only difference is about the time of reaction which for FAME it started and reached the maximum value sooner and also its value is much higher than $G$. Because $G$ was produced only by one reaction in spite of FAME by three parallel reactions. The results showed a satisfactory agreement with the experimental data by the maximum deviation of 0.8 and $0.57 \%$ at hydrodynamic and reaction sections, respectively. This simulation can be used for prediction of the reaction results, component concentration, optimum reactor length and optimum residual time. The last but not the least important point in some cases with no optimum size is CFD which can predict the best circulation time and material inlet rate.

\section{Acknowledgements}

The authors are grateful to the University of Malaya High Impact Research Grant (HIR-MOHE-D000038-16001) from the Ministry of Higher Education Malaysia and University of Malaya Bright Spark Unit, which financially supported this work. The authors would like to acknowledge Mahsa Ebrahim Moghaddam for English language editing.

\section{References}

[1] H. Falahati, A.Y. Tremblay, Fuel 91 (2012) 126.

[2] M.C.S. Gomes, P.A. Arroyo, N.C. Pereira, J. Membr. Sci. 378 (2011) 453.

[3] A.Y. Tremblay, P. Cao, M.A. Dubé, Energ. Fuel 22 (2008) 2748.

[4] J.M. Marchetti, V.U. Miguel, A.F. Errazu, Renew. Sust. Energ. Rev. 11 (2007) 1300

[5] M.A. Dasari, M.J. Goff, G.J. Suppes, JAOCS 80 (2003) 189.

[6] P.T. Vasudevan, M. Briggs, J. Ind. Microbiol. Biotechnol. 35 (2008) 421.

[7] X. Liu, H. He, Y. Wang, S. Zhu, X. Piao, Fuel 87 (2008) 216.

[8] I.M. Atadashi, M.K. Aroua, A.R. Abdul Aziz, N.M.N. Sulaiman, J. Membr. Sci. 15 (2012) 5051

[9] M.C.S. Gomes, N.C. Pereira, S.T.D.d. Barros, J. Membr. Sci. 352 (2010) 271.

[10] H. He, X. Guo, S. Zhu, J. Am. Oil Chem. Soc. 83 (2006) 457.

[11] J. Saleh, M.A. Dubé, A.Y. Tremblay, Energ. Fuel 24 (2010) 6179

[12] O. Kedem, A. Katchalsky, BBA 27 (1958) 229-246.

[13] S.H. Shuit, Y.T. Ong, K.T. Lee, B. Subhash, S.H. Tan, Biotechnol. Adv. 30 (2012) 1364

[14] G.S. Beavers, D.D. Joseph, J. Fluid Mech. 30 (1967) 197.

[15] S. Baroutian, M.K. Aroua, A.A.A. Raman, N.M.N. Sulaiman, Fuel Process. Technol. 91 (2010) 1378

[16] D. Darnoko, M. Cheryan, JAOCS 77 (2000) 1263.

[17] R.B. Bird, W.E. Stewart, E.N. Lightfoot, Transport Phenomena, 2nd ed., Wiley, New York, 2002.

[18] U. Shavit, G. Bar-Yosef, R. Rosenzweig, Water Resour. Res. 38 (2002) 1.

[19] Q. Liu, O.V. Vasilyev, J. Comput. Phys. 227 (2007) 946.

[20] T.A. Davis, ACM Trans. Math. Software 30 (2004) 196.

[21] T.A. Davis, I.S. Duff, ACM Trans. Math. Software 25 (1999) 1.

[22] O.C. Zeinkiewicz, R.L. Taylor, The Finite Element Method, 5th ed., Oxford Auckland Boston Johannes, Boston, 2000p. 128.

[23] S. Baroutian, M.K. Aroua, A.A.A. Raman, N.M.N. Sulaiman, Bioresour. Technol. 102 (2011) 1095

[24] L. Zhao, Novel Solid Base Catalysts for the Production of Biodiesel from Lipids, University of Kansas, Kansas, 2010.

[25] R.v.B. Aldert, Characterization of oil/water interfaces: a molecular dynamics study, Rijksuniversiteit Groningen, Vlaardingen, Nederland, 1995.

[26] W. Pronk, The Enzymatic Hydrolysis Of Lipids In A Hydrophilic Membrane Bioreactor, Landbouwuniversiteit, Wageningen, 1991.

[27] E. Richaud, B. Fayolle, B. Flaconnèche, J. Verdu, Biodiesel Permeability in Polyethylene, Ischia, Italy, 2012p. 170.

[28] G. Guevara-Carrion, C. Nieto-Draghi, J. Vrabec, H. Hasse, J. Phys. Chem. 112 (2008) 16664. 